Article

\title{
Effect of Tensor Correlations on the Density Dependence of the Nuclear Symmetry Energy
}

\author{
Isaac Vidaña ${ }^{1, *}$, Constança Providência ${ }^{1}$ and Artur Polls ${ }^{2}$ \\ ${ }^{1}$ Center of Computational Physics, Departement of Physics, University of Coimbra, \\ Coimbra PT-3004-516, Portugal; E-Mail: cp@ teor.fis.uc.pt \\ ${ }^{2}$ Departament d'Estructura i Constituents de la Matèria and Institut de Ciències del Cosmos, \\ Universitat de Barcelona, Avda. Diagonal 647, Barcelona ES-08028, Spain; \\ E-Mail: artur@ecm.ub.edu \\ * Author to whom correspondence should be addressed; E-Mail: ividana@ fis.uc.pt; \\ Tel.: +351-239-410-104.
}

Academic Editor: Dinesh Shetty

Received: 30 September 2014 / Accepted: 8 December 2014 / Published: 29 December 2014

\begin{abstract}
We analyze the effect of the tensor force and other components of the nucleon-nucleon interaction on the nuclear symmetry energy and its density dependence by using the Hellmann-Feynman theorem. The analysis is performed within the microscopic Brueckner-Hartree-Fock approach using the Argonne V18 potential plus a Urbana IX three-nucleon force. Our results show that the potential part of the nuclear Hamiltonian, and in particular its tensor component, gives the largest contribution to the symmetry energy. The decomposition of the symmetry energy into a kinetic part and a potential energy part provides physical insight on the correlated nature of the system, indicating that pure neutron matter is less correlated than symmetric nuclear matter.
\end{abstract}

Keywords: symmetry energy; tensor force; correlations

\section{Introduction}

The density dependence of the nuclear symmetry energy, $E_{\text {sym }}(\rho)$, is a crucial ingredient of the nuclear equation of state (EoS) needed to understand many important properties of isospin-rich nuclear systems such as exotic nuclei, supernovae and neutron stars [1-4]. Experimental information on 
$E_{\text {sym }}(\rho)$ below, close to, and above nuclear saturation density, $\rho_{0}$, can be obtained from a number of nuclear structure and heavy ion collision observables such as giant and pigmy resonances in heavy nuclei, $n / p$ and $t / 3 \mathrm{He}$ ratios in nuclear reactions, isospin diffusion and isospin scaling in nuclear multi-fragmentation, neutron-proton correlation functions at low relative momenta, isospin diffusion/transport in heavy-ion collisions, neutron-proton differential flow, isobaric analog states, or $\pi^{-} / \pi^{+}$and $K^{-} / K^{+}$ratios in heavy ion collisions. Accurate measurements of the neutron skin thickness $\delta R$ in heavy nuclei, via parity violating experiments or by means of antiprotonic atom data, can also help to constrain $E_{\text {sym }}(\rho)$, since its derivative is strongly correlated with $\delta R$. Additional information on $E_{\text {sym }}(\rho)$ can be extracted from the astrophysical observation of compact objects, which opens a window into both the bulk and the microscopic properties of nuclear matter at extreme isospin asymmetries. In particular, the characterization of the core-crust transition in neutron stars or the analysis of power-law correlations, such as the relation between the radius of a neutron star and the equation of state, can put stringent constraints on $E_{\text {sym }}(\rho)$.

Theoretically, $E_{\text {sym }}(\rho)$ has been determined using both phenomenological and microscopic many-body approaches. Phenomenological approaches, either relativistic or non-relativistic, are based on effective interactions that are frequently built to reproduce the properties of nuclei. Since many of such interactions are built to describe systems close to the symmetric case, predictions at high asymmetries should be, however, taken with care. Microscopic approaches start from realistic nucleon-nucleon (NN) interactions that reproduce the scattering and bound state properties of the free two-nucleon system and naturally include the isospin dependence. In-medium correlations are then built using many-body techniques that microscopically account for isospin asymmetry effects such as the difference in the Pauli blocking factors and self-energies of neutrons and protons in asymmetric matter.

Nevertheless, in spite of the experimental and theoretical efforts (see [1] and references therein) carried out in the last years and the significant recent progress in studying the properties of isospin-asymmetric nuclear systems, $E_{\text {sym }}(\rho)$ is still uncertain. Its value at saturation, $S_{0}$, is more or less well-established ( $\sim 30 \mathrm{MeV}$ ), and its behavior below saturation is now much better known [5]. However, for densities above saturation, $E_{\text {sym }}(\rho)$ is not yet well constrained, and the predictions from different models strongly diverge. Why $E_{\text {sym }}(\rho)$ is so uncertain is still an open question whose answer is related to our limited knowledge of the nuclear force, and in particular to its spin and isospin dependence.

In this work we analyze the effect of the tensor force and other components of the NN interaction on $E_{\text {sym }}(\rho)$, and discuss how the isospin dependence of the NN correlations affects it. This analysis is carried out with the help of the Hellmann-Feynman theorem [6,7] within the framework of the microscopic Brueckner-Hartree-Fock (BHF) approach [8,9]. This theorem allows the decomposition of the total energy per particle of both pure neutron matter (PNM) and symmetric nuclear matter (SNM), and therefore of the nuclear symmetry energy, in the kinetic and potential energy contributions. We explore the different effects of NN correlations on SNM and PNM and discuss how the isospin dependence of these correlations affects the density dependence of the nuclear symmetry energy. To such end we compare the kinetic and potential energy contributions with those of the free Fermi gas and the so-called correlation energy. We note that a similar analysis was already performed in [10,11]. Consequently, the present work can be considered a short review of these previous publications with a focus in some different aspects that provide new physical insights into the problem. We would like 
to point out that a more exhaustive and complete study requires the use of other nuclear Hamiltonians and many-body techniques. Particularly promising would be the use of chiral effective field theory interactions and Renormalization Group methods (see, e.g., [12-15] for recent developments). However, this is out of the scope of the present work. Such more exhaustive and complete study will eventually be considered in the future.

This paper is organized in the following way. In Section 2 we briefly review the BHF approach of asymmetric nuclear matter. Our results are presented in Section 3. Finally, a summary and our main conclusions are given in Section 4.

\section{The BHF Approach of Asymmetric Nuclear Matter}

Assuming charge symmetry for nuclear forces, the energy per particle of asymmetric nuclear matter can be well approximated as

$$
E(\rho, \beta) \sim E_{S N M}(\rho)+E_{\text {sym }}(\rho) \beta^{2}+\mathcal{O}(4)
$$

where $\beta=\left(\rho_{n}-\rho_{p}\right) /\left(\rho_{n}+\rho_{p}\right)$ is the isospin asymmetry parameter, $E_{S N M}(\rho)$ is the energy per particle of SNM and

$$
E_{\text {sym }}(\rho) \sim E(\rho, \beta=1)-E(\rho, \beta=0) \equiv E_{P N M}(\rho)-E_{S N M}(\rho)
$$

i.e., the difference of the energy per particle of PNM and SNM is a good approximation to the nuclear symmetry energy.

It is common to characterize the density dependence of the energy per particle of SNM around the saturation density $\rho_{0}$ in terms of a few bulk parameters by expanding it in a Taylor series around $\rho_{0}$,

$$
E_{S N M}(\rho)=E_{0}+\frac{K_{0}}{2}\left(\frac{\rho-\rho_{0}}{3 \rho_{0}}\right)^{2}+\frac{Q_{0}}{6}\left(\frac{\rho-\rho_{0}}{3 \rho_{0}}\right)^{3}+\mathcal{O}(4)
$$

The coefficients denote, respectively, the energy per particle, the incompressibility coefficient and the third derivative of the energy of SNM at saturation,

$$
E_{0}=E_{S N M}\left(\rho_{0}\right), \quad K_{0}=\left.9 \rho_{0}^{2} \frac{\partial^{2} E_{S N M}(\rho)}{\partial \rho^{2}}\right|_{\rho=\rho_{0}}, \quad Q_{0}=\left.27 \rho_{0}^{3} \frac{\partial^{3} E_{S N M}(\rho)}{\partial \rho^{3}}\right|_{\rho=\rho_{0}}
$$

Similarly, the behavior of the symmetry energy around saturation can also be characterized in terms of a few bulk parameters,

$$
E_{\text {sym }}(\rho)=S_{0}+L\left(\frac{\rho-\rho_{0}}{3 \rho_{0}}\right)+\frac{K_{\text {sym }}}{2}\left(\frac{\rho-\rho_{0}}{3 \rho_{0}}\right)^{2}+\frac{Q_{\text {sym }}}{6}\left(\frac{\rho-\rho_{0}}{3 \rho_{0}}\right)^{3}+\mathcal{O}(4)
$$

where $S_{0}$ is the value of the symmetry energy at saturation, and the quantities $L, K_{\text {sym }}$ and $Q_{s y m}$ are related to its slope, curvature and third derivative, respectively at such density,

$$
L=\left.3 \rho_{0} \frac{\partial E_{\text {sym }}(\rho)}{\partial \rho}\right|_{\rho=\rho_{0}}, \quad K_{\text {sym }}=\left.9 \rho_{0}^{2} \frac{\partial^{2} E_{\text {sym }}(\rho)}{\partial \rho^{2}}\right|_{\rho=\rho_{0}}, \quad Q_{\text {sym }}=\left.27 \rho_{0}^{3} \frac{\partial^{3} E_{\text {sym }}(\rho)}{\partial \rho^{3}}\right|_{\rho=\rho_{0}}
$$

The BHF approach is the lowest order of the Brueckner-Bethe-Goldstone many-body theory [8,9]. In this theory, the ground-state energy of nuclear matter is evaluated in terms of the so-called hole-line 
expansion, where the perturbative diagrams are grouped according to the number of independent hole lines. The expansion is derived by means of the in-medium two-body scattering $G$ matrix, which describes the effective interaction between two nucleons in the presence of a surrounding medium. It is obtained by solving the well-known Bethe-Goldstone equation, schematically written as

$$
G_{\tau_{1} \tau_{2} ; \tau_{3} \tau_{4}}(\omega)=V_{\tau_{1} \tau_{2} ; \tau_{3} \tau_{4}}+\sum_{i j} V_{\tau_{1} \tau_{2} ; \tau_{i} \tau_{j}} \frac{Q_{\tau_{i} \tau_{j}}}{\omega-\epsilon_{i}-\epsilon_{j}+i \eta} G_{\tau_{i} \tau_{j} ; \tau_{3} \tau_{4}}(\omega)
$$

where $\tau=n, p$ indicates the isospin projection of the two nucleons in the initial, intermediate and final states, $V$ denotes the bare NN interaction, $Q_{\tau_{i} \tau_{j}}$ denotes the Pauli operator that allows only intermediate states compatible with the Pauli principle, and $\omega$, the so-called starting energy, corresponds to the sum of non-relativistic energies of the interacting nucleons. The single-particle energy $\epsilon_{\tau}$ of a nucleon with momentum $\vec{k}$ is given by

$$
\epsilon_{\tau}(\vec{k})=\frac{\hbar^{2} k^{2}}{2 m_{\tau}}+\operatorname{Re}\left[U_{\tau}(\vec{k})\right]
$$

where the single-particle potential $U_{\tau}(\vec{k})$ represents the mean field "felt" by a nucleon due to its interaction with the other nucleons of the medium. In the BHF approximation $U_{\tau}(\vec{k})$ is calculated through the "on-shell energy" G-matrix and is given by

$$
U_{\tau}(\vec{k})=\sum_{\tau^{\prime}} \sum_{\left|\vec{k}^{\prime}\right|<k_{\tau_{\tau^{\prime}}}}\left\langle\vec{k} \vec{k}^{\prime}\left|G_{\tau \tau^{\prime} ; \tau \tau^{\prime}}\left(\omega=\epsilon_{\tau}(k)+\epsilon_{\tau^{\prime}}\left(k^{\prime}\right)\right)\right| \vec{k} \vec{k}^{\prime}\right\rangle_{A}
$$

where the sum runs over all neutron and proton occupied states and where the matrix elements are properly antisymmetrized. Once a self-consistent solution of Equations (7)-(9) is achieved, the energy per particle can be calculated as

$$
E_{B H F}(\rho, \beta)=\frac{1}{A} \sum_{\tau} \sum_{|\vec{k}|<k_{F_{\tau}}} \frac{\hbar^{2} k^{2}}{2 m_{\tau}}+\frac{1}{2 A} \sum_{\tau} \sum_{|\vec{k}|<k_{F_{\tau}}} \operatorname{Re}\left[U_{\tau}(\vec{k})\right]
$$

where the first term of the r.h.s. is simply the contribution of the free Fermi gas (FFG), and the second is the so-called correlation energy, $E_{\text {corr }}$. We note that $E_{B H F}$ represents only the sum of two-hole-line diagrams and includes only the effect of two-body correlations through the $G$ matrix. It has been shown by Song et al. $[16,17]$ that the contribution to the energy from three-hole-line diagrams (which accounts for the effect of three-body correlations) is minimized when the so-called continuous prescription [18] is adopted for the in-medium potential, which is a strong indication of the convergence of the hole-line expansion. We adopt this prescription in our BHF calculations with the Argonne V18 (Av18) NN potential [19] supplemented by the Urbana IX three-nucleon force (3NF) [20,21] which, for use in BHF calculations, was reduced to a two-body density-dependent force by averaging over the coordinates of the third nucleon in the medium [22-24]. The interested reader is referred to [25] for more details of our BHF calculation and to [26-28] for an extensive analysis of the use and effects of 3NFs in PNM and SNM.

\section{Results}

The discussion of our results starts by showing in Table 1 the bulk parameters characterizing the density dependence of the energy of SNM and the symmetry energy around saturation density. 
Results are shown for calculations with and without $3 \mathrm{NF}$. Note that, in general, the effect of the $3 \mathrm{NF}$ seems to be more important on the isoscalar properties than on the isovector ones. In fact, by looking at the table it seems that the parameters $L, K_{\text {sym }}$ and $Q_{\text {sym }}$ describing the density dependence of the symmetry energy around saturation are little affected by the effect of 3NF. However, we should notice that the value of the saturation density is different when $3 \mathrm{NFs}$ are considered $\left(\rho_{0}=0.187 \mathrm{fm}^{-3}\right)$ or not $\left(\rho_{0}=0.240 \mathrm{fm}^{-3}\right)$ in the calculation. Therefore, one has to be careful when comparing the value of these parameters in the two cases. To clarify the role of $3 \mathrm{NF}$ in the isovector properties of the nuclear equation of state, we show in Figure 1 the energy per particle of PNM (panel a) and SNM (panel b), as well as the symmetry energy (panel c) and its slope parameter (panel d), defined as $L(\rho)=3 \rho \partial E_{\text {sym }}(\rho) / \partial \rho$, as a function of the density. The circles show the results at saturation density. As expected in the low density regime the role of $3 \mathrm{NFs}$ is basically negligible. Overall, for densities in $0.1-0.3 \mathrm{fm}^{-3}$, there is a moderate dependence of $E_{\text {sym }}(\rho)$ and $L(\rho)$ on the $3 \mathrm{NF}$, even though its effect on PNM and SNM is not small. This dependence becomes stronger and stronger when increasing the density. The importance of 3 NFs has been recently revised in the context of chiral effective field theory [29-34]. New fitting protocols of two-nucleon forces seem to indicate that the effect of $3 \mathrm{NF}$ could actually be rather small in PNM. Nevertheless, the relative importance of the two- and three-body contributions changes with the resolution scale. It is not clear whether such observations, valid for somewhat soft chiral interactions, still apply when considering a harder interaction like Av18.

Table 1. Bulk parameters characterizing the density dependence of the energy per particle of SNM and the symmetry energy around the saturation density for our BHF calculation with and without $3 \mathrm{NF}$. All quantities are in $\mathrm{MeV}$, except $\rho_{0}$, given in $\mathrm{fm}^{-3}$.

\begin{tabular}{ccccccccc}
\hline Calculation & $\boldsymbol{\rho}_{\mathbf{0}}$ & $\boldsymbol{E}_{\mathbf{0}}$ & $\boldsymbol{K}_{\mathbf{0}}$ & $\boldsymbol{Q}_{\mathbf{0}}$ & $\boldsymbol{E}_{\text {sym }}$ & $\boldsymbol{L}$ & $\boldsymbol{K}_{\text {sym }}$ & $\boldsymbol{Q}_{\boldsymbol{s y m}}$ \\
\hline BHF (3NF) & 0.187 & -15.23 & 195.5 & -280.9 & 34.3 & 66.5 & -31.3 & -112.8 \\
BHF (no 3NF) & 0.240 & -17.30 & 213.6 & -225.1 & 35.8 & 63.1 & -27.8 & -159.8 \\
\hline
\end{tabular}

In Figure 2 we show the free Fermi gas and correlation energy contributions to the energy per particle of both PNM (panel a) and SNM (panel b) as well as to the symmetry energy (panel c) and its slope parameter (panel d) as a function of the density obtained in our BHF calculation with Av18 + 3NF. The particular values of these contributions at the saturation density of our calculation $\rho_{0}=0.187 \mathrm{fm}^{-3}$ are denoted by circles and are reported in Table 2. The symmetry energy is calculated, as mentioned before, as the difference of the total energy per particle of PNM and SNM. As it is seen in the figure (see also Figure 4) the FFG energy of PNM is always larger than the corresponding one of SNM and, therefore, its contribution to the symmetry energy is positive throughout the explored density range and, in particular, amounts to $\sim 14.38 \mathrm{MeV}$ at $\rho_{0}$. The correlation energy, $E_{\text {corr }}$, contributes also positively $\left(\sim 19.92 \mathrm{MeV}\right.$ at $\left.\rho_{0}\right)$ to the symmetry energy for all the densities, since it gives smaller attraction in PNM than in SNM. This contribution is larger than that of the FFG. The addition of both contributions, which are of the same order at $\rho_{0}$, provides a symmetry energy of $\sim 34.3 \mathrm{MeV}$ at this density. The contributions to $L$ can be decomposed similarly. In this case the contribution of the correlation energy is much larger than that of the FFG, except at very low densities where both contributions are similar. 

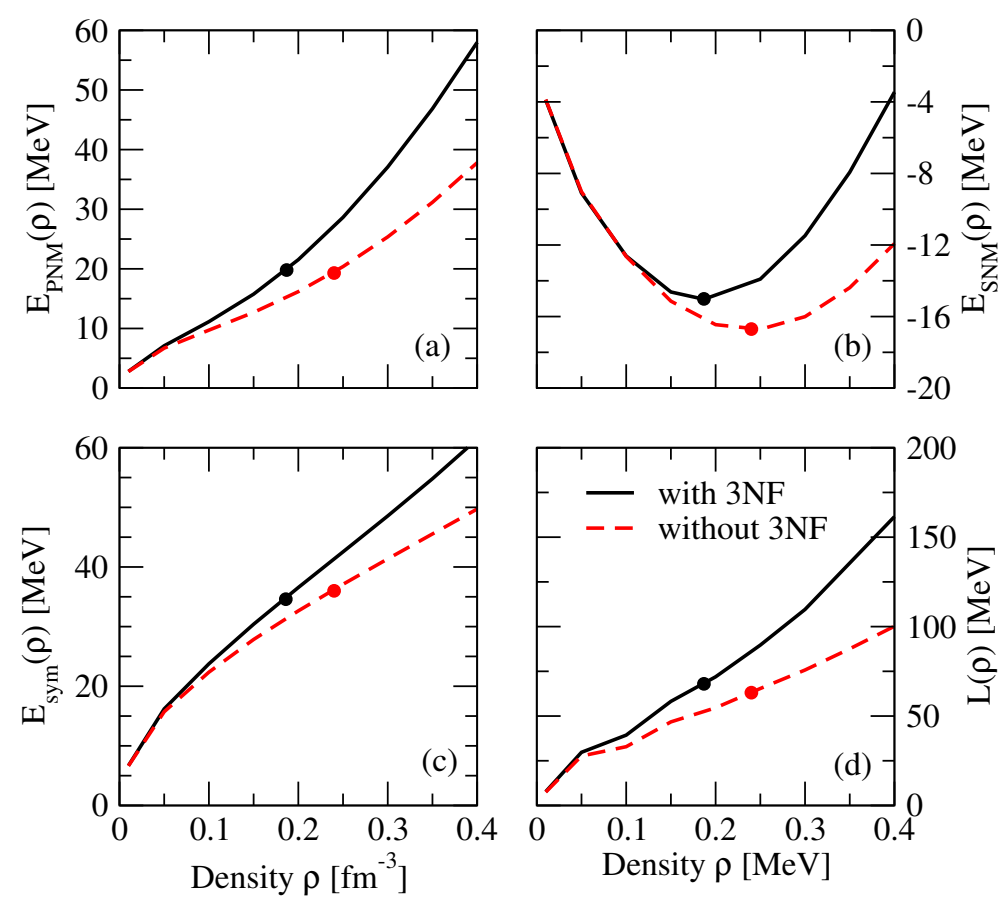

Figure 1. (Color on-line) Energy per particle of PNM (a) and SNM (b) as a function of the density. Density dependence of the symmetry energy (c) and its slope parameter $L(\mathbf{d})$. The results have been obtained in our BHF calculation with (solid lines) and without (dashed lines) $3 \mathrm{NF}$. The circles show the result at saturation density.
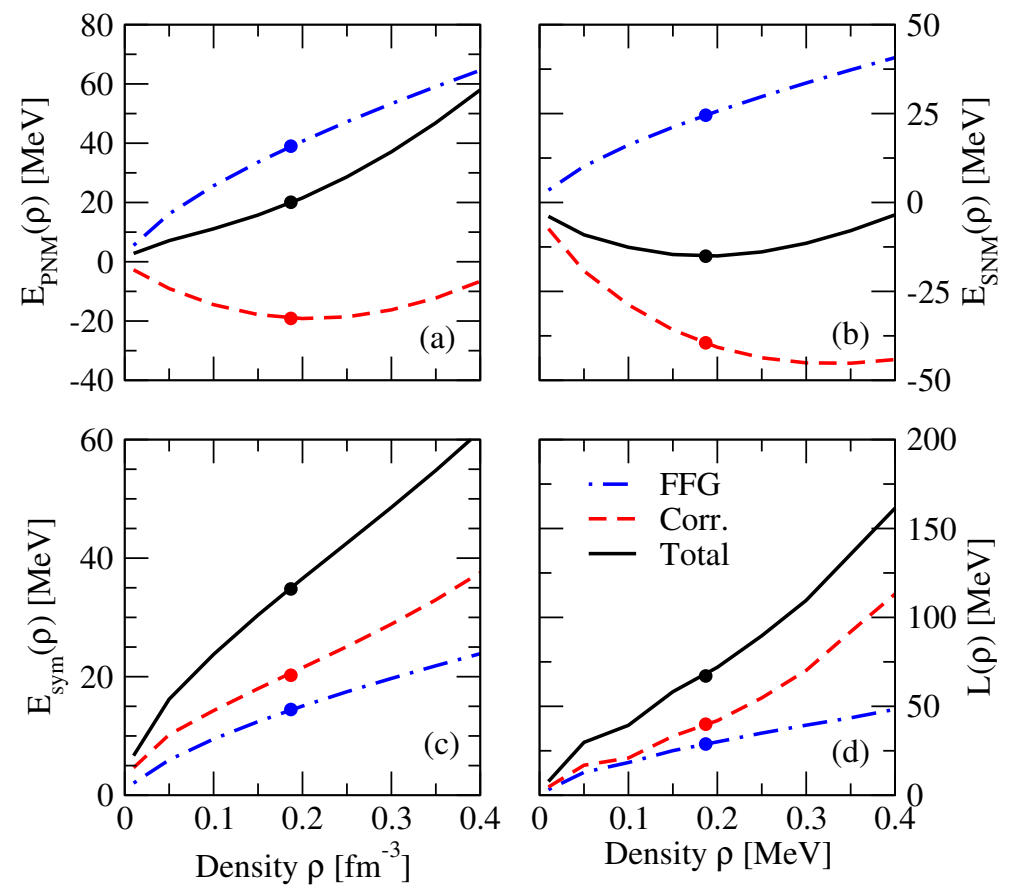

Figure 2. (Color on-line) Free Fermi gas and correlation energy contributions to the total energy per particle of PNM (a) and SNM (b), and to symmetry energy (c) and its slope parameter (d), as a function of density. Results are shown for our BHF calculation with Av18 +3 NF. The circles show the result at saturation density. 
Table 2. Free Fermi gas and correlation energy contributions to the total energy per particle of PNM and SNM at the saturation density $\rho_{0}=0.187 \mathrm{fm}^{-3}$ of our BHF calculation with Av18 +3 NF. The corresponding contributions to the symmetry energy $E_{\text {sym }}$ and its slope parameter $L$ are also shown. All results are given in $\mathrm{MeV}$.

\begin{tabular}{ccccc}
\hline Contribution & $\boldsymbol{E}_{\boldsymbol{P N M}}$ & $\boldsymbol{E}_{\boldsymbol{S N M}}$ & $\boldsymbol{E}_{\text {sym }}$ & $\boldsymbol{L}$ \\
\hline$E_{F F G}$ & 38.991 & 24.529 & 14.462 & 28.779 \\
$E_{\text {corr }}$ & -19.921 & -39.759 & 19.838 & 37.721 \\
\hline Total & 19.070 & -15.230 & 34.300 & 66.500 \\
\hline
\end{tabular}

To get a further physical insight on the role of the correlation energy, it is useful to look at its spin-isospin $(S, T)$ decomposition, shown in Table 3. As expected, the main contribution is that of the $(1,0)$ channel, which is acting only in SNM and has a large attractive contribution. It is precisely in this channel where the tensor component of the nuclear force is active. Notice that the $T=1$ channels give similar contributions in PNM and SNM and, therefore, the contribution to the symmetry energy is small. The channel $(0,0)$ gives a repulsive contribution to the total energy in SNM and since it does not play any role in PNM, its contribution to the total symmetry energy is negative. Note again that the tensor force is not acting in this channel.

Table 3. Spin-isospin $(S, T)$ channel decomposition to the correlation energy of PNM and SNM at the saturation density $\rho_{0}=0.187 \mathrm{fm}^{-3}$ of our BHF calculation with Av18 $+3 \mathrm{NF}$. The decomposition of correlation energy contribution to the symmetry energy and its slope parameter is also shown. All results are given in $\mathrm{MeV}$.

\begin{tabular}{ccccc}
\hline$(\boldsymbol{S}, \boldsymbol{T})$ & $\boldsymbol{E}_{\text {corr }}^{\boldsymbol{P N M}}$ & $\boldsymbol{E}_{\text {corr }}^{\boldsymbol{S N M}}$ & $\boldsymbol{E}_{\text {corr }}^{\text {sym }}$ & $\boldsymbol{L}_{\text {corr }}$ \\
\hline$(0,0)$ & 0 & 5.894 & -5.894 & -23.085 \\
$(0,1)$ & -21.280 & -17.923 & -3.357 & -3.142 \\
$(1,0)$ & 0 & -28.363 & 28.363 & 51.696 \\
$(1,1)$ & 1.359 & 0.633 & 0.726 & 12.252 \\
\hline
\end{tabular}

Let us further continue this analysis by looking at the contributions of the different partial waves to $E_{\text {corr }}$. Contributions up to $J=8$ have been considered. These contributions are shown in Table 4 . Notice that the ${ }^{1} S_{0}$ contribution, which is dominated by the central component of the NN potential, has a similar large contribution to both PNM and SNM and, therefore, its effect on the symmetry energy is almost negligible. The largest contribution is provided by the ${ }^{3} S_{1}-{ }^{3} D_{1}$ channel, which corresponds to $T=0$, active only in SNM. For larger values of the total angular momentum $J$, the contributions become smaller and many cancellations take place. In general, one observes that the final energy in SNM is the result of a large cancellation between $E_{F F G}$ and $E_{c o r r}$, and that the absolute value of $E_{\text {corr }}$ for PNM is significantly smaller than for SNM. These observations point to the well-accepted fact that neutron matter is less correlated than symmetric matter. 
Table 4. Partial wave decomposition of the correlation energy of PNM and SNM at the saturation density $\rho_{0}=0.187 \mathrm{fm}^{-3}$ of our BHF calculation with Av18 $+3 \mathrm{NF}$. The decomposition of correlation energy contribution to the symmetry energy and its slope parameter is also shown. All results are given in $\mathrm{MeV}$.

\begin{tabular}{ccccc}
\hline Partial Wave & $\boldsymbol{E}_{\text {corr }}^{\boldsymbol{P N M}}$ & $\boldsymbol{E}_{\text {corr }}^{\boldsymbol{S N M}}$ & $\boldsymbol{E}_{\text {corr }}^{\text {sym }}$ & $\boldsymbol{L}_{\text {corr }}$ \\
\hline${ }^{1} S_{0}$ & -14.330 & -14.407 & 0.077 & 11.229 \\
${ }^{3} S_{1}$ & 0 & -24.865 & 24.865 & 35.521 \\
${ }^{1} P_{1}$ & 0 & 5.193 & -5.193 & -20.201 \\
${ }^{3} P_{0}$ & -4.522 & -3.713 & -0.809 & 0.224 \\
${ }^{3} P_{1}$ & 18.459 & 12.002 & 6.457 & 27.702 \\
${ }^{3} P_{2}$ & -13.550 & -8.102 & -5.448 & -17.784 \\
${ }^{1} D_{2}$ & -5.850 & -3.154 & -2.696 & -10.888 \\
${ }^{3} D_{1}$ & 0 & 1.036 & -1.036 & -3.894 \\
${ }^{3} D_{2}$ & 0 & -3.795 & 3.795 & 15.844 \\
${ }^{3} D_{3}$ & 0 & -0.522 & 0.522 & 3.305 \\
${ }^{1} F_{3}$ & 0 & 0.699 & -0.699 & -3.394 \\
${ }^{3} F_{2}$ & -0.651 & -0.221 & -0.430 & -1.515 \\
${ }^{3} F_{3}$ & 2.022 & 0.826 & 1.196 & 5.026 \\
${ }^{3} F_{4}$ & -0.743 & -0.183 & -0.560 & -3.006 \\
${ }^{1} G_{4}$ & -0.810 & -0.247 & -0.563 & 3.029 \\
${ }^{3} G_{3}$ & 0 & 0.002 & -0.002 & 0.425 \\
${ }^{3} G_{4}$ & 0 & -0.213 & 0.213 & 0.449 \\
${ }^{3} G_{5}$ & 0 & -0.053 & 0.053 & 0.617 \\
${ }^{1} H_{5}$ & 0 & 0.029 & -0.029 & 0.122 \\
${ }^{3} H_{4}$ & 0.034 & 0.040 & -0.007 & 0.224 \\
${ }^{3} H_{5}$ & 0.226 & -0.33 & 0.258 & 0.949 \\
${ }^{3} H_{6}$ & 0.044 & 0.035 & 0.010 & 0.136 \\
Rest up to $J=8$ & -0.220 & 0.219 & -0.439 & -6.399 \\
\hline${ }^{3}$ & & & &
\end{tabular}

By using different phase shift equivalent NN potentials, it has been shown that the total binding energy of the deuteron is the result of a strong cancellation between the kinetic and the potential energy [35,36]. However, this observation should be taken with care when using softer modern low-momentum or chiral $\mathrm{NN}$ interactions, since in this case this cancellation may be not so strong. The large kinetic energy, in the deuteron case, is a consequence of the NN correlations existing in the ${ }^{3} S_{1}-{ }^{3} D_{1}$ channel. In that sense we would like to study the decomposition of the total energy of the infinite system in the kinetic and potential energy. Due to NN correlations, the kinetic energy will be larger than the FFG energy. Therefore, the difference between the kinetic energy of a correlated nuclear system and that of a FFG can be used to quantify the effect of NN correlations.

Unfortunately, the BHF approach does not give direct access to the separate contributions of the kinetic and potentials energies because it does not provide the correlated many-body wave function, $|\Psi\rangle$. However, it has been shown [37-40] that the Hellmann-Feynman theorem [6,7] can be used to estimate the ground-state expectation values of both contributions from the derivative of the total energy with respect to a properly introduced parameter. Writing the nuclear matter Hamiltonian as $H=T+V$, and 
defining a $\lambda$-dependent Hamiltonian $H(\lambda)=T+\lambda V$, the expectation value of the potential energy is given as

$$
\langle V\rangle \equiv \frac{\langle\Psi|V| \Psi\rangle}{\langle\Psi \mid \Psi\rangle}=\left(\frac{d E}{d \lambda}\right)_{(\lambda=1)}
$$

Then, the kinetic energy contribution $\langle T\rangle$ can be simply obtained by subtracting $\langle V\rangle$ from the total energy $E$.

In Figure 3 we show the kinetic and potential energy contributions to the energy per particle of both PNM (panel a) and SNM (panel b) as well as to the symmetry energy (panel c) and its slope parameter (panel d) as a function of the density obtained by applying the Hellmann-Feynman theorem, as explained above, to our BHF calculation with Av18 + 3NF. The values of all these contributions at saturation density are denoted by circles and reported in Table 5.
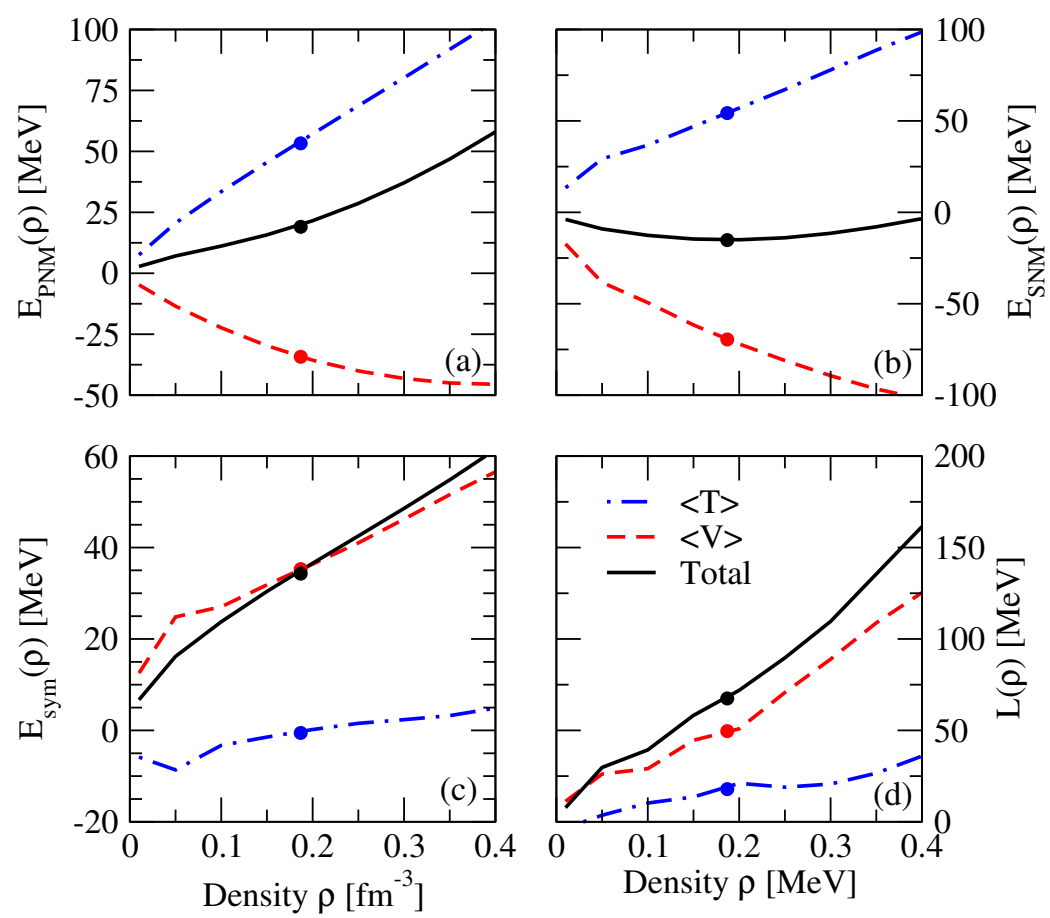

Figure 3. (Color on-line) Kinetic $\langle T\rangle$ and potential $\langle V\rangle$ energy contributions to the total energy per particle of PNM (a) and SNM (b) and to symmetry energy (c) and its slope parameter (d) as a function of density. Results are shown for our BHF calculation with Av18 +3 NF. The circles show the results at saturation density.

Table 5. Kinetic, $\langle T\rangle$, and potential, $\langle V\rangle$, energy contributions to the total energy per particle of PNM and SNM at the saturation density $\rho_{0}=0.187 \mathrm{fm}^{-3}$ of our BHF calculation with Av18 +3 NF. The corresponding contributions to the symmetry energy $E_{\text {sym }}$ and its slope parameter $L$ are also shown. All results are given in $\mathrm{MeV}$.

\begin{tabular}{ccccc}
\hline Contribution & $\boldsymbol{E}_{\boldsymbol{P N} \boldsymbol{M}}$ & $\boldsymbol{E}_{\boldsymbol{S N M}}$ & $\boldsymbol{E}_{\boldsymbol{s y m}}$ & $\boldsymbol{L}$ \\
\hline$\langle T\rangle$ & 53.321 & 54.294 & -0.973 & 14.896 \\
$\langle V\rangle$ & -34.251 & -69.524 & 35.273 & 51.604 \\
\hline Total & 19.070 & -15.230 & 34.300 & 66.500 \\
\hline
\end{tabular}


As in the case of the deuteron, both the total energy of PNM and that of SNM are the result of a strong cancellation between the kinetic and potential energies. It is worth noticing that the kinetic energy contribution to $E_{\text {sym }}$ is very small in the whole density range, being slightly negative for $\rho \leq 0.2 \mathrm{fm}^{-3}$. This is in contrast to the results for the FFG (see Figure 2 and Table 2). The increase of the kinetic energy with respect to the FFG energy is probably due to the strong isospin dependence of the short-range NN correlations (SRC) induced by the tensor force. This observation has been also recently pointed out by $\mathrm{Xu}$ and $\mathrm{Li}$ [41] and Hen et al. [42] using a phenomenological model for the nucleon momentum distribution $n(k)$, and by Carbone et al. [43] using the self-consistent Green's function approach with the Av18 and the CDBONN [44] potentials. To illustrate this point, we show in Figure 4 the kinetic energy of uncorrelated (i.e., FFG energy) and correlated PNM and SNM as a function of density. Results at saturation density are also shown in this case by the circles. It can be seen that the increase of the kinetic energy of SNM due to SRC is always much larger than that of PNM. This is an indication that, at the same fixed density, SNM is always more correlated than PNM. Note also that the kinetic energy of both correlated systems is of similar order and that their strong cancellation gives the almost negligible contribution to the kinetic part of $E_{\text {sym }}$ noticed before. It is also worth mentioning that the kinetic contribution to $L$ is smaller than the corresponding one of the FFG in the full density range. Clearly, the major contribution to both the symmetry energy and its slope parameter is due to the potential energy part. Note that at saturation density (see Table 5) this contribution is practically equal to the total value of the symmetry energy at saturation, and it represents $\sim 78 \%$ of $\mathrm{L}$.

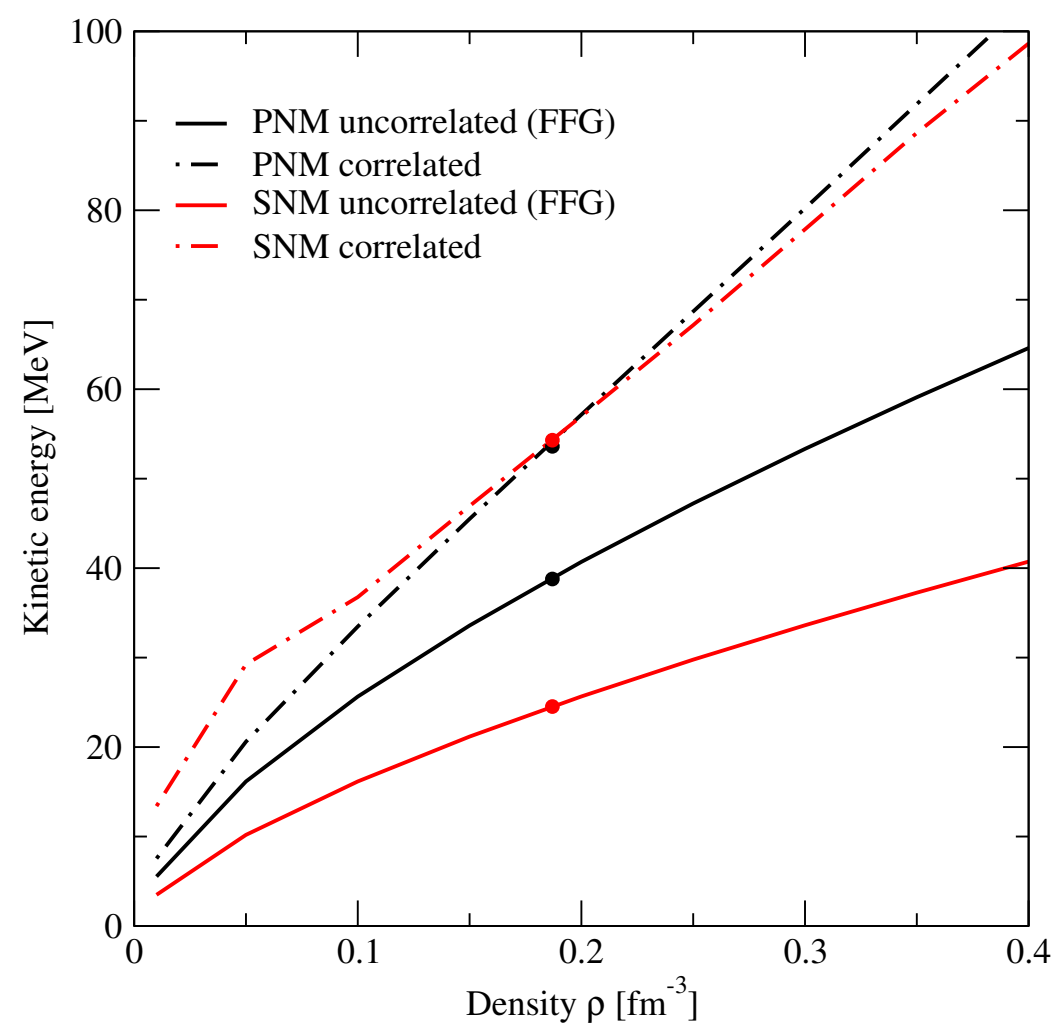

Figure 4. (Color on-line) Kinetic energy per particle of uncorrelated (i.e., FFG) and correlated PNM and SNM as a function of density. The circles show the result at saturation density. 
The spin-isospin $(S, T)$ channel decomposition of the potential energy of PNM and SNM at saturation, as well as of its contribution to $E_{\text {sym }}$ and $L$, is also illustrative. This is reported in Table 6 . As in the case of the correlation energy, the largest contribution to the potential part of both $E_{\text {sym }}$ and $L$ is provided by the $(S=1, T=0)$ channel, for which the tensor is active. Interestingly, the $S=0$ channels have a smaller and similar negative contribution to $E_{\text {sym }}$ and also a moderate negative contribution to $L$. However, the origin of these contributions is qualitatively different. While the channel $(S=0, T=0)$ does not contribute to PNM and gives a small repulsive contribution to SNM, the contribution of the channel $(S=0, T=1)$ to $E_{\text {sym }}$ is the result of a strong cancellation of large attractive contributions of this channel in both PNM and SNM. Analogous conclusions can be obtained from Table 7, where the partial wave decomposition of the potential energy is reported. Note that similar arguments have been already pointed out by other authors [41-43,45-57].

Table 6. Spin-isospin $(S, T)$ channel decomposition to the potential energy of PNM and SNM at the saturation density $\rho_{0}=0.187 \mathrm{fm}^{-3}$ of our BHF calculation with Av18 $+3 \mathrm{NF}$. The decomposition of potential energy contribution to the symmetry energy and its slope parameter is also shown. All results are given in $\mathrm{MeV}$.

\begin{tabular}{ccccc}
\hline$(\boldsymbol{S}, \boldsymbol{T})$ & $\langle\boldsymbol{V}\rangle_{\boldsymbol{P N M}}$ & $\langle\boldsymbol{V}\rangle_{\boldsymbol{S N M}}$ & $\boldsymbol{E}_{\langle\boldsymbol{V}\rangle}^{\text {sym }}$ & $\boldsymbol{L}_{\langle\boldsymbol{V}\rangle}$ \\
\hline$(0,0)$ & 0 & 5.600 & -5.600 & -21.457 \\
$(0,1)$ & -29.889 & -23.064 & -6.825 & -17.950 \\
$(1,0)$ & 0 & -49.836 & 49.836 & 90.561 \\
$(1,1)$ & -4.3621 & -2.224 & -2.138 & 0.450 \\
\hline
\end{tabular}

Finally, we analyze the role played by the different terms of the nuclear force, particularly the one of the tensor force, in the determination of $E_{\text {sym }}$ and $L$. To such end, we apply the Hellmann-Feynman theorem to the separate components of the Av18 and the Urbana IX 3NF. The Av18 potential has 18 components of the form $v_{p}\left(r_{i j}\right) O_{i j}^{p}$ with

$$
\begin{aligned}
O_{i j}^{p=1,18=} & 1, \vec{\tau}_{i} \cdot \vec{\tau}_{j}, \vec{\sigma}_{i} \cdot \vec{\sigma}_{j},\left(\vec{\sigma}_{i} \cdot \vec{\sigma}_{j}\right)\left(\vec{\tau}_{i} \cdot \vec{\tau}_{j}\right), S_{i j}, S_{i j}\left(\vec{\tau}_{i} \cdot \vec{\tau}_{j}\right), \vec{L} \cdot \vec{S}, \vec{L} \cdot \vec{S}\left(\vec{\tau}_{i} \cdot \vec{\tau}_{j}\right), L^{2}, \\
& L^{2}\left(\vec{\tau}_{i} \cdot \vec{\tau}_{j}\right), L^{2}\left(\vec{\sigma}_{i} \cdot \vec{\sigma}_{j}\right), L^{2}\left(\vec{\sigma}_{i} \cdot \vec{\sigma}_{j}\right)\left(\vec{\tau}_{i} \cdot \vec{\tau}_{j}\right),(\vec{L} \cdot \vec{S})^{2},(\vec{L} \cdot \vec{S})^{2}\left(\vec{\tau}_{i} \cdot \vec{\tau}_{j}\right), \\
& T_{i j},\left(\vec{\sigma}_{i} \cdot \vec{\sigma}_{j}\right) T_{i j}, S_{i j} T_{i j},\left(\tau_{z i}+\tau_{z_{j}}\right)
\end{aligned}
$$

being $S_{i j}$ the usual tensor operator; $\vec{L}$ the relative orbital angular momentum; $\vec{S}$ the total spin of the nucleon pair; and $T_{i j}=3 \tau_{z i} \tau_{z j}-\vec{\tau}_{i} \cdot \vec{\tau}_{j}$ the isotensor operator. Note that the last four operators break the charge independence of the nuclear interaction.

As we said above, the Urbana IX three-body force is reduced to an effective density-dependent two-body force when used in the BHF approach. For simplicity, in the following we refer to it as the reduced Urbana force. This force is made of three components of the type $u_{p}\left(r_{i j}, \rho\right) O_{i j}^{p}$ where

$$
O_{i j}^{p=1,3}=1,\left(\vec{\sigma}_{i} \cdot \vec{\sigma}_{j}\right)\left(\vec{\tau}_{i} \cdot \vec{\tau}_{j}\right), S_{i j}\left(\vec{\tau}_{i} \cdot \vec{\tau}_{j}\right)
$$

introducing additional central, $\sigma \tau$ and tensor terms (see, e.g., [24] for details).

The separate contributions of the various components of the Av18 potential and the reduced Urbana force to the energy per particle of PNM and SNM, and to the symmetry energy and its slope parameter 
at the saturation density, are given in Table 8. The contribution from the tensor component to $E_{\text {sym }}$ and $L$ (contributions $\left\langle V_{S_{i j}}\right\rangle$ and $\left\langle V_{S_{i j}\left(\vec{\tau}_{i} \cdot \vec{\tau}_{j}\right)}\right\rangle$ from the Av18 potential, and $\left\langle U_{S_{i j}\left(\vec{\tau}_{i} \cdot \vec{\tau}_{j}\right)}\right\rangle$ from the reduced Urbana force) is $36.056 \mathrm{MeV}$ and $69.968 \mathrm{MeV}$, respectively. These results clearly confirm that the tensor force gives the largest contribution to both $E_{\text {sym }}$ and $L$. The contributions from the other components either are negligible, as for instance the contribution from the charge symmetry breaking terms $\left(\left\langle V_{T_{i j}}\right\rangle\right.$, $\left\langle V_{\left(\vec{\sigma}_{i} \cdot \vec{\sigma}_{j}\right) T_{i j}}\right\rangle,\left\langle V_{S_{i j} T_{i j}}\right\rangle$ and $\left.\left\langle V_{\left(\tau_{z i}+\tau_{z_{j}}\right)}\right\rangle\right)$, or almost cancel out.

Table 7. Partial wave decomposition of the potential energy of PNM and SNM at the saturation density $\rho_{0}=0.187 \mathrm{fm}^{-3}$ of our BHF calculation with Av18 $+3 \mathrm{NF}$. The decomposition of potential energy contribution to the symmetry energy and its slope parameter is also shown. All results are given in $\mathrm{MeV}$.

\begin{tabular}{ccccc}
\hline Partial Wave & $\langle\boldsymbol{V}\rangle_{\boldsymbol{P N} \boldsymbol{M}}$ & $\langle\boldsymbol{V}\rangle_{\boldsymbol{S N} \boldsymbol{M}}$ & $\boldsymbol{E}_{\langle\boldsymbol{V}\rangle}^{\boldsymbol{s y \boldsymbol { m }}}$ & $\boldsymbol{L}_{\langle\boldsymbol{V}\rangle}$ \\
\hline${ }^{1} S_{0}$ & -23.070 & -19.660 & -3.410 & -3.459 \\
${ }^{3} S_{1}$ & 0 & -45.810 & 45.810 & 71.855 \\
${ }^{1} P_{1}$ & 0 & 4.904 & -4.904 & -18.601 \\
${ }^{3} P_{0}$ & -5.321 & -4.029 & -1.292 & -1.898 \\
${ }^{3} P_{1}$ & 16.110 & 10.720 & 5.390 & 21.949 \\
${ }^{3} P_{2}$ & -16.000 & -9.334 & -6.666 & -21.168 \\
${ }^{1} D_{2}$ & -5.956 & -3.201 & -2.755 & -11.033 \\
${ }^{3} D_{1}$ & 0 & 0.981 & -0.981 & -3.739 \\
${ }^{3} D_{2}$ & 0 & -3.982 & 3.982 & 16.601 \\
${ }^{3} D_{3}$ & 0 & -0.798 & 0.798 & 4.895 \\
${ }^{1} F_{3}$ & 0 & 0.694 & -0.694 & -3.348 \\
${ }^{3} F_{2}$ & -0.695 & -0.229 & -0.466 & -1.799 \\
${ }^{3} F_{3}$ & 2.000 & 0.821 & 1.179 & 4.883 \\
${ }^{3} F_{4}$ & -0.796 & -0.194 & -0.602 & -3.239 \\
${ }^{1} G_{4}$ & -0.812 & -0.247 & -0.565 & -3.036 \\
${ }^{3} G_{3}$ & 0 & -0.001 & 0.001 & 0.441 \\
${ }^{3} G_{4}$ & 0 & -0.213 & 0.213 & 0.449 \\
${ }^{3} G_{5}$ & 0 & -0.057 & 0.057 & 0.650 \\
${ }^{1} H_{5}$ & 0 & 0.029 & -0.029 & 0.107 \\
${ }^{3} H_{4}$ & 0.033 & 0.040 & -0.007 & 0.232 \\
${ }^{3} H_{5}$ & 0.225 & -0.033 & 0.258 & 0.968 \\
${ }^{3} H_{6}$ & 0.043 & 0.034 & 0.009 & 0.144 \\
Rest up to $J=8$ & -0.012 & 0.041 & -0.161 & -0.250 \\
\hline${ }^{3}$ & & & &
\end{tabular}


Table 8. Contributions of the various components of the Av18 potential (denoted as $\left\langle V_{i}\right\rangle$ ) and the reduced Urbana force (denoted as $\left\langle U_{i}\right\rangle$ ) to the energy per particle of PNM and SNM and to the symmetry energy and its slope parameter at the saturation density $\rho_{0}=0.187 \mathrm{fm}^{-3}$. Units are given in $\mathrm{MeV}$.

\begin{tabular}{ccccc}
\hline Component & $\langle\boldsymbol{V}\rangle_{\boldsymbol{P N M}}$ & $\langle\boldsymbol{V}\rangle_{\boldsymbol{S N M}}$ & $\boldsymbol{E}_{\langle\boldsymbol{V}\rangle}^{\text {sym }}$ & $\boldsymbol{L}_{\langle\boldsymbol{V}\rangle}$ \\
\hline$\left\langle V_{1}\right\rangle$ & -31.212 & -32.710 & 1.498 & -5.580 \\
$\left\langle V_{\vec{\tau}_{i} \cdot \vec{\tau}_{j}}\right\rangle$ & -4.957 & 3.997 & -8.954 & -20.383 \\
$\left\langle V_{\vec{\sigma}_{i} \cdot \vec{\sigma}_{j}}\right\rangle$ & -0.319 & -0.382 & 0.063 & 2.392 \\
$\left\langle V_{\left(\vec{\sigma}_{i} \cdot \vec{\sigma}_{j}\right)\left(\vec{\tau}_{i} \cdot \vec{\tau}_{j}\right)}\right\rangle$ & -5.724 & -11.388 & 5.664 & 2.521 \\
$\left\langle V_{S_{i j}}\right\rangle$ & -0.792 & 1.912 & -2.704 & -4.998 \\
$\left\langle V_{S_{i j}\left(\vec{\tau}_{i} \cdot \vec{\tau}_{j}\right)}\right\rangle$ & -4.989 & -37.592 & 32.603 & 47.095 \\
$\left\langle V_{\vec{L} \cdot \vec{S}}\right\rangle$ & -7.538 & -1.754 & -5.784 & -12.251 \\
$\left\langle V_{\vec{L} \cdot \vec{S}\left(\vec{\tau}_{i} \cdot \vec{\tau}_{j}\right)}\right\rangle$ & -2.671 & -6.539 & 3.868 & 3.969 \\
$\left\langle V_{L^{2}}\right\rangle$ & 11.850 & 13.610 & -1.760 & 1.521 \\
$\left\langle V_{L^{2}\left(\vec{\tau}_{i} \cdot \vec{\tau}_{j}\right)}\right\rangle$ & -2.788 & 0.270 & -3.058 & -14.262 \\
$\left\langle V_{L^{2}\left(\vec{\sigma}_{i} \cdot \vec{\sigma}_{j}\right)}\right\rangle$ & 1.265 & 1.383 & -0.118 & 1.405 \\
$\left\langle V_{L^{2}\left(\vec{\sigma}_{i} \cdot \vec{\sigma}_{j}\right)\left(\vec{\tau}_{i} \cdot \vec{\tau}_{j}\right)}\right\rangle$ & 0.051 & 0.008 & 0.043 & -0.341 \\
$\left\langle V_{(\vec{L} \cdot \vec{S})^{2}}\right\rangle$ & 4.194 & 5.682 & -1.488 & -0.327 \\
$\left\langle V_{\left(\vec{L} \cdot \vec{S}^{2}\left(\vec{\tau}_{i} \cdot \vec{\tau}_{j}\right)\right.}\right\rangle$ & 5.169 & -6.190 & 11.359 & 31.368 \\
$\left\langle V_{T_{i j}}\right\rangle$ & 0.003 & 0.039 & -0.036 & -0.022 \\
$\left\langle V_{\left(\vec{\sigma}_{i} \cdot \vec{\sigma}_{j}\right) T_{i j}}\right\rangle$ & -0.017 & -0.106 & 0.089 & 0.042 \\
$\left\langle V_{S_{i j} T_{i j}}\right\rangle$ & 0.004 & 0.079 & -0.075 & -0.124 \\
$\left\langle V_{\left(\tau_{z_{i}}+\tau_{z_{j}}\right)}\right\rangle$ & -0.084 & -0.001 & -0.083 & -0.331 \\
$\left\langle U_{1}\right\rangle$ & 2.985 & 3.251 & -0.266 & -0.630 \\
$\left\langle U_{\left(\vec{\sigma}_{i} \cdot \vec{\sigma}_{j}\right)\left(\vec{\tau}_{i} \cdot \vec{\tau}_{j}\right)}\right\rangle$ & 2.254 & 3.999 & -1.745 & -7.228 \\
$\left\langle U_{S_{i j}\left(\vec{\tau}_{i} \cdot \vec{\tau}_{j}\right)}\right\rangle$ & -0.935 & -7.092 & 6.157 & 27.768 \\
\hline & & & &
\end{tabular}

\section{Summary and Conclusions}

In this work we have analyzed the effect of the tensor force and other components of the nucleon-nucleon (NN) interaction on the nuclear symmetry energy and its slope parameter for a wide range of densities. We have also discussed the role of the isospin dependence of the NN correlations on both quantities. This analysis has been carried out with the help of the Hellmann-Feynman theorem within the framework of the microscopic Brueckner-Hartree-Fock (BHF) approach using the Av18 potential plus an effective density-dependent two-body force deduced from the Urbana IX three-body force. The theorem allowed the decomposition of the total energy per particle of PNM and SNM into their kinetic and potential energy contributions. Our results showed that the potential part of the nuclear Hamiltonian gives the main contribution to both $E_{\text {sym }}$ and $L$. The kinetic contribution to $E_{\text {sym }}$ is very small throughout the explored density range, being even slightly negative for $\rho \leq 0.2 \mathrm{fm}^{-3}$. We have explored the different effects of NN correlations on SNM and PNM and discussed how the isospin dependence of these correlations affects the density dependence of the nuclear symmetry energy. To such end, we have compared the kinetic and potential energy contributions to the energy per particle of PNM and SNM and to $E_{\text {sym }}(\rho)$ and $L(\rho)$ with those of the free Fermi gas and the correlation energy. We found that PNM is less correlated than SNM, in agreement with recent results [41-43]. Finally, we have 
performed a partial wave and a spin-isospin channel decomposition of the potential part of $E_{\text {sym }}$ and $L$, showing that the major contribution to them is given by the spin-triplet $(S=1)$ and isospin-singlet $(T=0)$ channel. As we have explicitly shown, this is due to the dominant effect of the tensor force, which gives the largest contribution to both $E_{\text {sym }}$ and $L$. In conclusion, our results confirm the critical role of the tensor force in the determination of the symmetry energy and its density dependence. We would like to finish by noticing that at present there are no experimental or theoretical constraints to control the role of the tensor forces beyond the saturation density, although there are indications, based on large $N_{c}$ QCD arguments (see, e.g., [58-60]), that tensor forces could undergo non-trivial modifications at densities not too far above saturation density, due to either the topology changes in the nucleon structure or the emergence of strongly interacting quark degrees of freedom. Consequently, the reader should take our results above saturation density with care.

\section{Acknowledgments}

This work has been partially supported by the project PEst-OE/FIS/UI0405/2014 developed under the initiative QREN financed by the UE/FEDER through the program COMPETE-"Programa Operacional Factores de Competitividade", by the grant No. FIS2011-24154 from MICINNC (Spain) and grant No. 2014SGR401 from Generalitat de Catalunya (Spain), by the Consolider Ingenio 2010 Programme CPAN CSD2007-0042; STFC, through grants ST/1005528/1 and ST/J000051/1, and "NewCompstar", COST Action MP1304.

\section{Author Contributions}

Isaac Vidaña was responsible for the calculations and the writting of the manuscript; Artur Polls and Constança Providência contributed to the writting of the manuscript.

\section{Conflicts of Interest}

The authors declare no conflict of interest.

\section{References}

1. Li, B.A.; Ramos, A.; Verde, G.; Vidaña, I. Topical issue on nuclear symmetry energy. Eur. Phys. J. A 2014, 50, doi:10.1140/epja/i2014-14009-x.

2. Baran, V.; Colonna, M.; Greco, V.; di Toro, M. Reaction dynamics with exotic nuclei. Phys. Rep. 2005, 410, 335-466.

3. Li, B.A.; Chen, W.; Ko, C.M. Recent progress and new challenges in isospin physics with heavy-ion reactions. Phys. Rep. 2008, 464, 113-281.

4. Steiner, A.W.; Prakash, M.; Lattimer, J.; Ellis, P.J. Isospin asymmetry in nuclei and neutron stars. Phys. Rep. 2005, 411, 325-375.

5. Tsang, M.B.; Stone, J.R.; Camera, F.; Danielewicz, P.; Gandolfi, S.; Hebeler, K.; Horowitz, C.J.; Lee, J.; Lynch, W.G.; Kohley, Z.; et al. Constraints on the symmetry energy and neutron skins from experiments and theory. Phys. Rev. C 2012, 86, doi:10.1103/PhysRevC.86.015803. 
6. Hellmann, H. Einfüring in Die Quantenchemie; Franz Deuticke: Leipzig, Germany, 1937; p. 285.

7. Feynman, R.P. Forces in molecules. Phys. Rev. 1936, 56, 340-343.

8. Day, B.D. Elements of Brueckner-Goldstone theory of nuclear matter. Rev. Mod. Phys. 1967, 39, 719-744.

9. Baldo, M. Nuclear Methods and the Nuclear Equation of State; World Scientific: Singapore, 1999.

10. Vidaña, I.; Polls, A.; Providência, C. Nuclear symmetry energy and the role of the tensor force. Phys. Rev. C 2011, 84, doi:10.1103/PhysRevC.84.062801.

11. Carbone, A.; Polls, A.; Providência, C.; Rios, A.; Vidaña, I. Tensor force effects and high-momentum components in the nuclear symmetry energy. Eur. Phys. J. A 2014, 50, doi:10.1140/epja/i2014-14013-2.

12. Hagen, G.; Papenbrock, T.; Ekström, A.; Wendt, K.A.; Baardsen, G.; Gandolfi, S.; Hjorth-Jensen, M.; Horowitz, C.J. Coupled-cluster calculations of nucleonic matter. Phys. Rev. C 2014, 89, doi:10.1103/PhysRevC.89.014319.

13. Holt, J.W.; Kaiser, N.; Weise, W. Nuclear chiral dynamics and thermodynamics. Prog. Part. Nucl. Phys. 2013, 73, 35-83.

14. Furnstahl, R.J.; Hebeler, K. New applications of renormalization group methods in nuclear physics. Rept. Prog. Phys. 2013, 76, doi:10.1088/0034-4885/76/12/126301.

15. Hebeler, K.; Bogner, S.K.; Furnstahl, R.J.; Nogga, A.; Schwenk, A. Improved nuclear matter calculations from chiral low-momentum interactions. Phys. Rev. C 2011, 83, doi:10.1103/ PhysRevC.83.031301.

16. Song, H.Q.; Baldo, M.; Giansiracusa, G.; Lombardo, U. Bethe-Brueckner-Goldstone expansion in nuclear matter Phys. Rev. Lett. 1998, 81, 1584-1587.

17. Song, H.Q.; Baldo, M.; Giansiracusa, G.; Lombardo, U. Three-hole line contribution in nuclear matter revisited. Phys. Lett. B 1999, 411, 237-243.

18. Jekeune, J.P.; Lejeune, A.; Mahaux, C. Many-body theory of nuclear matter. Phys. Rep. 1976, 25, 83-174.

19. Wiringa, R.B.; Stoks, V.G.J.; Schiavilla, R. Accurate nucleon-nucleon potential with charge-independence breaking. Phys. Rev. C 1995, 51, 38-51.

20. Pudliner, B.S.; Pandharipande, V.R.; Carlson, J.; Wiringa, R.B. Quantum Monte Carlo calculations of $A \leq 6$ nuclei. Phys. Rev. Lett. 1995, 74, 4396-4399.

21. Pudliner, B.S.; Pandharipande, V.R.; Carlson, J.; Pieper, S.C.; Wiringa, R.B. Quantum Monte Carlo calculations of nuclei with $A \leq 7$. Phys. Rev. $C$ 1997, 56, 1720-1750.

22. Loiseau, B.A.; Nogami, Y.; Ross, C.K. Nucleon-nucleon correlation and two-pion exchange three-body force in nuclear matter. Nucl. Phys. A 1971, 165, 601-624.

23. Grangé, P.; Martzolff, M.; Nogami, Y.; Sprung, D.W.L.; Ross, C.K. Three-body force in nuclear matter. Phys. Lett. B 1976, 60, 237-240.

24. Baldo, M.; Ferreira, L. Nuclear liquid-gas phase transition. Phys. Rev. C 1999, 59, 682-703.

25. Vidaña, I.; Providência, C.; Polls, A.; Rios, A. Density dependence of the nuclear symmetry energy: A microscopic perspective. Phys. Rev. C 2009, 80, doi:10.1103/PhysRevC.80.045806.

26. Zhou, X.R.; Burgio, G.F.; Lombardo, U.; Schulze, H.-J.; Zuo, W. Three-body forces and neutron star structure. Phys. Rev. C 2004, 69, doi:10.1103/PhysRevC.69.018801. 
27. Li, Z.H.; Lombardo, U.; Schulze, H.-J.; Zuo, W. Consistent nucleon-nucleon potentials and three-body forces. Phys. Rev. C 2008, 77, doi:10.1103/PhysRevC.77.034316.

28. Li, Z.H.; Schulze, H.-J. Neutron star structure with modern nucleonic three-body forces. Phys. Rev. C 2008, 78, doi:10.1103/PhysRevC.78.028801.

29. Epelbaum, E.; Hammer, H.-W.; Meißner, U.-G. Modern theory of nuclear forces. Rev. Mod. Phys. 2009, 81, 1773-1825.

30. Machleidt, R.; Entem, D.R. Chiral effective field theory and nuclear forces. Phys. Rep. 2011, 503, 1-75.

31. Hammer, H.-W.; Nogga, A.; Schwenk, A. Three-body forces: From cold atoms to nuclei. Rev. Mod. Phys. 2013, 85, doi:10.1103/RevModPhys.85.197.

32. Carbone, A.; Polls, A.; Rios, A. Symmetric nuclear matter with chiral three-nucleon forces in the self-consistent Green's functions approach. Phys. Rev. C 2013, 88, doi:10.1103/PhysRevC.88.044302.

33. Carbone, A.; Cipollone, A.; Barbieri, C.; Rios, A.; Polls, A. Self-consistent Green's function formalism with three-body interactions. Phys. Rev. C 2013, 88, doi:10.1103/PhysRevC.88.054326.

34. Ekström, A.; Baardsen, G.; ForssÃl'n, C.; Hagen, G.; Hjorth-Jensen, M.; Jansen, G.R.; Machleidt, R.; Nazarewicz, W.; Papenbrock, T.; Sarich, J.; et al. Optimized chiral nucleon-nucleon interaction at next-to-next-to-leading order. Phys. Rev. Lett. 2013, 110, doi:10.1103/PhysRevLett.110.192502.

35. Polls, A.; Müther, H; Machleidt, R.; Hjorth-Jensen, M. Phaseshift equivalent NN potentials and the deuteron. Phys. Lett. B 1998, 432, 1-7.

36. Baldo, M.; Polls, A.; Rios, A.; Schulze, H.-J.; Vidaña, I. Comparative study of neutron and nuclear matterwith simplified Argonne nucleon-nucleon potentials. Phys. Rev. C 2012, 86, doi:10.1103/PhysRevC.86.064001.

37. Müther, H.; Polls, A. Correlations derived from modern nucleon-nucleon potentials. Phys. Rev. C 1999, 61, doi:10.1103/PhysRevC.61.014304.

38. Sartor, R. Diagrammatic analysis of the Hellmann-Feynman theorem. Phys. Rev. C 2000, 62, doi:10.1103/PhysRevC.62.044318.

39. Sartor, R. Diagrammatic analysis of the Hellmann-Feynman theorem: Numerical applications. Phys. Rev. C 2001, 64, doi:10.1103/PhysRevC.64.054303.

40. Bombaci, I.; Fabrocini, A.; Polls, A.; Vidaña, I. Spin-orbit and tensor interactons in homogeneous matter of nucleons: Accuracy of many-body theories. Phys. Lett. B 2005, 609, 232-240.

41. Xu, C.; Li, B.A. Tensor force induced isospin-dependence of short-range nucleon-nucleon correlation and high density behavious of nuclear symmetry energy. 2011. Available online: http://arxiv.org/abs/1104.2075 (accessed on 8 December 2014).

42. Hen, O.; Li, B.A.; Guo, W.-J.; Weinstein, L.B.; Piasetzky, E. Kinetic symmetry energy of nucleonic matter with tensor correlations. 2014. Available online: http://arxiv.org/abs/1408.0772 (accessed on 8 December 2014).

43. Carbone, A.; Polls, A.; Rios, A. High momentum components in the nuclear symmetry energy. EuroPhys. Lett. 2012, 97, doi:10.1209/0295-5075/97/22001. 
44. Machleidt, R.; Sammarruca, F.; Song, Y. Nonlocal nature of the nuclear force and its impact on nuclear structure. Phys. Rev. C 1996, 53, R1483-R1487.

45. Bombaci, I.; Lombardo, U. Asymmetric nuclear matter equation of state. Phys. Rev. C 1991, 44, 1892-1900.

46. Engvik, L.; Hjorth-Jensen, M.; Machleidt, R.; Müther, H.; Polls, A. Modern nucleon-nucleon potentials and symmetry energy in infinite matter. Nucl. Phys. A 1997, 627, 85-100.

47. Pandharipande, V.R.; Garde, V.K. Effect of tensor forces on composition of dense matter. Phys. Lett. B 1972, 39, 608-610.

48. Wiringa, R.B.; Fiks, V.; Fabrocini, A. Equation of state for dense nucleon matter. Phys. Rev. C 1988, 38, 1010-1037.

49. Brown, G.E.; Rho, M. In-medium stiffening of the nucleon-nucleon spin-isospin interaction. Phys. Lett. B 1990, 237, 3-7.

50. Müther, H.; Prakash, M.; Ainsworth, T.L. The nuclear symmetry energy in relativistic Brueckner-Hartree-Fock calculations. Phys. Lett. B 1987, 199, 469-574.

51. Zuo, W.; Bombaci, I.; Lombardo, U. Asymmetric nuclear matter from an extended Brueckner-Hartree-Fock appriach. Phys. Rev. C 1999, 60, doi:10.1103/PhysRevC.60.024605.

52. Zuo, W.; Lejeune, A.; Lombardo, U.; Mathiot, J.F. Microscopic three-body force for asymmetric nuclear matter. Eur. Phys. J. A 2002, 14, 469-475.

53. Dieperink, A.E.L.; Dewulf, Y.; van Neck, D.; Waroquier, M.; Rodin, V. Nuclear symmetry energy and the neutron skin in neutron-rich nuclei. Phys. Rev. C 2003, 68, doi:10.1103/ PhysRevC.68.064307.

54. Li, Z.H.; Lombardo, U.; Schulze, H.-J.; Zuo, W.; Chen, L.W.; Ma, H.R. Nuclear matter saturation point and symmetry enegy with modern nucleon-nucleon potentials. Phys. Rev. C 2006, 74, doi:10.1103/PhysRevC.74.047304.

55. Xu, C.; Li, B.A. Understanding the major uncertainties in the nuclear symmetry energy at suprasaturation densities. Phys. Rev. C 2010, 81, doi:10.1103/PhysRevC.81.064612.

56. Li, A.; Li, B.A. Short-range tensor interaction and high-density nuclear symmetry energy. 2011. Available online: http://arxiv.org/abs/1107.0496 (accessed on 8 December 2014).

57. Sammarruca, F. Contribution of isovector mesons to the symmetry energy in a microscopic model. Phys. Rev. C 2011, 84, doi:10.1103/PhysRevC.84.044307.

58. Kyu, H.; Rho, M. Topology change and tensor forces for the EoS of dense baryonic matter. Eur. Phys. J. A 2014, 50, doi:10.1140/epja/i2014-14014-1.

59. Seo, Y.; Sin, S.-J. Symmetry energy from holographic QCD. Eur. Phys. J. A 2014, 50, doi:10.1140/epja/i2014-14015-0.

60. Sang, K.; Lee, S.H. Symmetry energy from QCD sum rules. Eur. Phys. J. A 2014, 50, doi:10.1140/epja/i2014-14016-y.

(c) 2014 by the authors; licensee MDPI, Basel, Switzerland. This article is an open access article distributed under the terms and conditions of the Creative Commons Attribution license (http://creativecommons.org/licenses/by/4.0/). 